\title{
Correlation between the expression of Drp1 in vascular endothelial cells and inflammatory factors in hypertension rats
}

\author{
XINGHUI LIU*, HONGWEN TAN*, XIAOQIAO LIU and QIANG WU \\ Department of Cardiology, Guizhou Provincial People's Hospital, Guiyang, Guizhou 550002, P.R. China
}

Received November 8, 2017; Accepted January 4, 2018

DOI: $10.3892 /$ etm.2018.5899

\begin{abstract}
The objective of this study was to investigate the expression level of dynamin-related protein 1 (Drp1) in vascular endothelium of hypertension rats and its correlation with expression of inflammatory factors. Twenty spontaneous hypertension rats (SHR) were randomly divided into SHR group $(n=10)$ and inhibition group (MD group, $n=10)$, and the Sprague Dawley rats were enrolled as the control group ( $\mathrm{C}$ group, $\mathrm{n}=10)$. For rats in the MD group, Mdivi-1, a mitochondrial division inhibitor, was given in dosage of $25 \mathrm{mg} / \mathrm{kg}$. After 4 weeks of administration, blood pressure was measured via tail-artery blood pressure measurement. The blood samples collected from the abdominal aorta of rats were used to assay the $\mathrm{C}$-reaction protein (CRP) concentration in serum through radioimmunoassay. Hematoxylin and eosin (H\&E) staining was performed for sections of thoracic aorta for morphological observation and measurement of medial thickness. Enzymelinked immunosorbant assay (ELISA), semi-quantitative real-time polymerase chain reaction (RT-PCR) and western blotting was carried out for detecting the expression levels of interleukin-6 (IL-6) and tumor necrosis factor- $\alpha$ (TNF- $\alpha$ ). Drp1 and monocyte chemotactic protein 1 (MCP-1). After 4 weeks of drug administration, the blood pressure in the MD group was significantly higher $(\mathrm{P}<0.01)$. The medial thickness of the thoracic aorta in the MD group was significantly decreased in comparison with the SHR group $(\mathrm{P}<0.01)$. The results of ELISA showed that compared with the SHR group, the expression levels of IL-6 and TNF- $\alpha$ in the MD group were remarkably decreased $(\mathrm{P}<0.01)$. Semi-quantitative RT-PCR results indicated that the mRNA expression levels of Drp1 and MCP-1 in the MD group were significantly lower than those in the SHR group $(\mathrm{P}<0.05)$. In the SHR rats, after administration of Mdivi-1, the expression of Drp1 is decreased, which
\end{abstract}

Correspondence to: Dr Qiang Wu, Department of Cardiology, Guizhou Provincial People's Hospital, 83 Zhongshan East Road, Guiyang, Guizhou 550002, P.R. China

E-mail: guiywu@sina.com

${ }^{*}$ Contributed equally

Key words: spontaneous hypertension rat, dynamin-related protein 1 , inflammatory factors, vessels contributes to the alleviation in inflammatory reactions and protects the vessels in SHR rats.

\section{Introduction}

With the increase in arterial pressure, hypertension is a disease usually accompanied by severe complications in vascular system of heart, brain and kidney, severely threatening the life and health of human beings. As the life quality has been continuously improved, an increasing trend on a year-by-year basis has been identified in the incidence rate of cardiovascular diseases; in addition, the incidence rate of complications induced by hypertension is gradually increased; considering all these factors, researchers have paid great attention to the pathogenesis of hypertension and vascular lesions (1-3). In recent years, although the pathogenesis and pathological process of hypertension remains poorly understood, literature has reported that damaged vascular endothelial cells, thickened vascular wall and non-specific inflammatory responses are the major factors contributing to the onset of hypertension (4-6). Dynaminrelated protein 1 (Drp1) plays a key role in the regulation of mitochondrial division; according to the study of $\mathrm{Hu}$ et al (7), they found that mitochondrial fusion/division imbalance greatly contributes to the ischemia-reperfusion injuries and sepsis, in which Drp1 has a regulatory role (8). Tanwar et al (9) indicated that inhibition on Drp1 can block cell apoptosis caused by the tumor necrosis factor. However, there is no literature reporting the role of Drp1 in hypertension and its effect on inflammatory factors. In this study, we investigated how variations induced by inhibition of Drp1 in inflammatory responses in endothelial cells of hypertension could ameliorate the vessels.

Spontaneous hypertension rat (SHR) models were established for exploring the variations caused by Drp1 in vascular endothelial cells, the effect of Drp1 on inflammatory factors, and the effect of inhibition on Drp1 on inflammatory factors in vascular endothelial cells and the vessels in spontaneous hypertension, aiming to provide new ideas for elucidating the pathogenesis of spontaneous hypertension and developing effective clinical treatment methods.

\section{Materials and methods}

Instruments and materials. Mdivi-1 (Selleck, Houston, TX, USA); TRIzol kit, rat anti-Drp1 antibody, enzyme-linked immunosorbent assay (ELISA) kit for interleukin-6 (IL-6) and 
ELISA kit for tumor necrosis factor- $\alpha$ (TNF- $\alpha$ ) (all from BD Biosciences, Franklin Lakes, NJ, USA); secondary antibody against rat (Beijing Zhongshan Golden Bridge Biotech Co., Ltd., Beijing, China); rabbit anti-IL-6 (1:500; cat. no. 12153), rabbit anti-TNF- $\alpha$ (1:500; cat. no. 8184), rabbit anti-MCP-1 (1:500; cat. no. 39091), horseradish peroxidase-labeled secondary antibody against rabbit $(1: 1,000$; cat. no. 7074$)$ (all from Cell Signaling Technology Co., Ltd.); electrochemiluminescence (ECL) solution and color development powder (both from Invitrogen, Carlsbad, CA, USA); pipette (Eppendorf, Hamburg, Germany); electronic scale (BP121S; Sartorious, Goettingen, Germany); $-80^{\circ} \mathrm{C}$ refrigerator and lowtemperature centrifuge (Thermo Fisher Scientific, Dreieich, Germany); other instruments and reagents are indicated in the corresponding part in this study.

Experimental animals and grouping. In this study, we selected a total of 10 male, healthy, Sprague-Dawley rats and 20 spontaneous hypertension rats (SHR) with the weight ranging from 220 to $250 \mathrm{~g}$. All these animals were purchased from Guangdong Medical Laboratory Animal Center, and the qualification number of experimental animals was SCXK (Guangdong) 2013-0015. The 20 SHR rats were randomly divided into two groups, i.e. the SHR group $(n=10)$ and the inhibition group (MD group; $n=10$ ). Rats in the MD group received Mdivi-1 (25 mg/kg) every day, while rats in the normal control group (C group) and the SHR group were given normal saline $(10 \mathrm{ml} / \mathrm{kg} /$ day $)$ every day. The study was approved by the Ethics Committee of Guizhou Provincial People's Hospital (Guiyang, China).

\section{Sample collection and preparation}

Preparation of the serum samples. After 4 weeks of drug administration, blood was drawn from the abdominal aorta of rats in each group after the blood pressure and weight had been measured. Blood samples were then centrifuged at $2060 \mathrm{x} \mathrm{g}$ for $15 \mathrm{~min}$, and the supernatant was divided into several pieces and placed into Eppendorf (EP) tubes. The EP tubes were at $-80^{\circ} \mathrm{C}$.

Preparation of abdominal aorta. After blood collection, the thoracic artery at $0.5 \mathrm{~cm}$ below the aortic arch was immediately isolated, some of which were then preserved at $-80^{\circ} \mathrm{C}$, while the remaining artery was rinsed with Hanks solution and fixed in $4 \%$ paraformaldehyde. For the resected thoracic aorta, routine paraffin embedding, serial sectioning and hematoxylin eosin staining were sequentially performed followed by observation of variations in vascular media under the microscope, in which 5 visions were randomly selected for each rat, and the images were loaded into the IPP 6.0 image analytic system to measure the medial thickness of the vascular wall.

Detection of $C$-reaction protein $(C R P)$ content in serum. Radioimmunoassay (RIA) was performed to assay the content of CRP in serum. The serum samples that were centrifuged and frozen were preserved at $-20^{\circ} \mathrm{C}$, and then thawed at room temperature. In the numbered polystyrene tubes, $100 \mu 1$ ${ }^{125} \mathrm{I}$-CRP and standard substance of CRP were added and mixed well. Then the tubes were transferred into a dark room for incubation at $37^{\circ} \mathrm{C}$ for $1 \mathrm{~h}$. Thereafter, $500 \mu \mathrm{l}$ separating
Table I. Primers for PCR.

Gene Primer sequence

MCP-1 F: 5'-TGTCCCAAAGAAGCTGTAGTATTTGT-3' R: 5'-TTCTGATCTCACTTGGTTCTGTCC-3'

Drp1 F: 5'-GGAATCTTCTTCATTCCTGAC-3' R: 5'-CCAGTGCAGGGTCCGAGGT-3'

GAPDH F: 5'-GATGATTGGCATGGCTTT-3' R: 5'-CACCTTCCGTTCCAGTTT-3'

F, forward, R, reverse.

agent was dropped into the tubes that were later shaken and placed at room temperature for $20 \mathrm{~min}$. Then the tubes were centrifuged at $2800 \times \mathrm{g}$ for $15 \mathrm{~min}$, and the supernatant was discarded. Standard curve was prepared with the counts per minute as the vertical coordinate and the corresponding standard substance of CRP as the horizontal coordinate, and, accordingly, the concentration of CRP in serum samples of each rat in all groups was calculated.

Detection of content of inflammatory factors in serum via ELISA. Procedures were carried out in strict accordance with the instruction of ELISA kit: standard substances of interleukin- 6 and tumor necrosis factor- $\alpha$ were prepared for assay of standard curve. In each well, $100 \mu \mathrm{l}$ sample or standard substance was added for $90 \mathrm{~min}$ of reaction at $37^{\circ} \mathrm{C}$ after the plate was sealed using a membrane. Then, we added the $100 \mu 1$ biotin-labeled anti-rat TNF- $\alpha$ and IL- 6 antibodies for $60 \mathrm{~min}$ of reaction at $37^{\circ} \mathrm{C}$. Then on the plate, $300 \mu \mathrm{l}$ washing buffer was added, and after the mixture was soaked for $1 \mathrm{~min}$, the buffer was discarded. In each well, $90 \mu$ l color development solution was added, and the plate was sealed using membrane and placed in the dark for $30 \mathrm{~min}$ of reaction at $37^{\circ} \mathrm{C}$. Thereafter, $100 \mu 1$ termination solution was added, and the color of solution turned from blue to yellow. With a microplate reader, the optical density in each well at wavelength of $450 \mathrm{~nm}$ was detected, and accordingly, the standard curve was prepared using CurveExpert 1.4 software. Then the concentration of inflammatory factors in each well was calculated, and the actual concentration of inflammatory factors in the sample was the production result and the dilution ratio.

Detection of mRNA levels of Drpl and MCP-1 via semi-quantitative polymerase chain reaction $(P C R)$. RNA in serum of rats in all groups was extracted with TRIzol kit to measure the $D_{260} /$ $\mathrm{D}_{280}$ ratio, and the results suggested that ratio was between 1.8 and 2.0. The RNA integrity was confirmed through agarose gel electrophoresis, and the results showed that at $28 \mathrm{~S}, 18 \mathrm{~S}$ and $5 \mathrm{~S}$, and the brightness of he stripe at $28 \mathrm{~S}$ was approximately twice of that at $18 \mathrm{~S}$. All these results indicated that the RNA was integral and could be used in following experiments. Reverse transcription was performed for preparation of cDNA using the relevant kit, and with GAPDH as internal reference, the mRNA expression levels of MCP-1 and CCR-2 were detected using semi-quantitative PCR in following reaction conditions: initial 
A

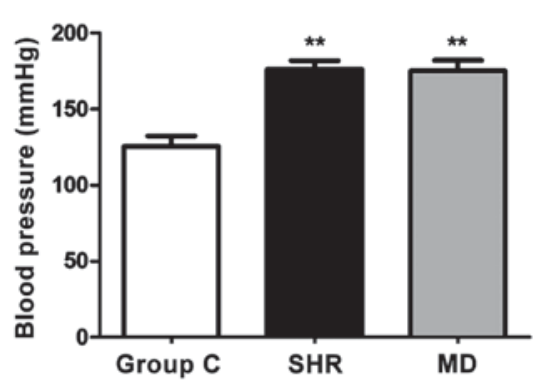

B

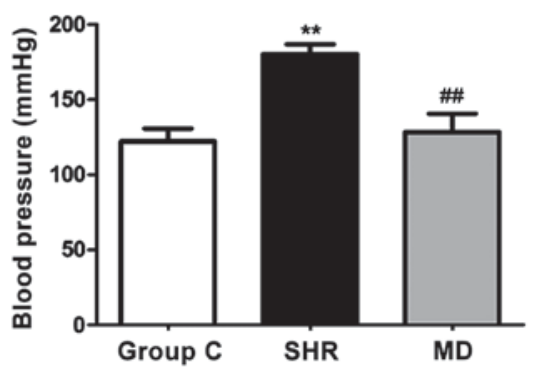

Figure 1. Variation in blood pressure of rats in each group. (A) Blood pressure of rats in each group before drug administration. The blood pressure of rats in the spontaneous hypertension rat (SHR) group and the MD group is significantly higher than that in the $\mathrm{C}$ group, and the difference has statistical significance $\left({ }^{* *} \mathrm{P}<0.01\right)$. (B) After 4 weeks of drug administration, variations in blood pressure of rats in each group. The blood pressure of rats in the MD group is significantly lower than that in the SHR group $\left({ }^{\# \#} \mathrm{P}<0.01\right)$.

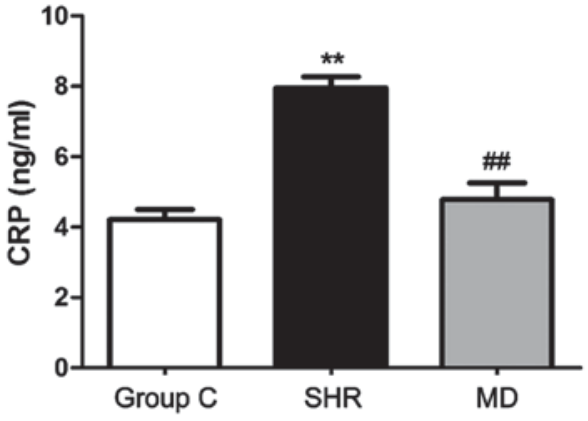

Figure 2. Variation in C-reaction protein (CRP) levels in serum of rats in each group: The concentration of CRP in serum of rats in the $\mathrm{C}$ group is significantly lower than those in the spontaneous hypertension rat (SHR) group and the MD group $\left({ }^{* *} \mathrm{P}<0.01\right)$; the level of CRP in serum of rats in the MD group is lower than that in the SHR group, and the difference has statistical significance $\left.{ }^{\# \#} \mathrm{P}<0.01\right)$.

denaturation at $95^{\circ} \mathrm{C}$ for $5 \mathrm{~min} ; 95^{\circ} \mathrm{C}$ for $30 \mathrm{sec}, 64^{\circ} \mathrm{C}$ for $25 \mathrm{sec}$, $72^{\circ} \mathrm{C}$ for $30 \mathrm{sec}$, for a total of 35 cycles; extension at $72^{\circ} \mathrm{C}$ for $5 \mathrm{~min}$. Primers were synthesized by Tiangen Biotech (Beijing) Co., Ltd. (Beijing, China), and the sequences are shown in Table I. After reaction, agarose gel electrophoresis was performed for the products followed by observation through ultraviolet imaging system. The mRNA expression levels of MCP-1 and Drp1 in each group are indicated by MCP-1/ GAPDH and Drp1/GAPDH ratios.

Detection of the expression levels of relevant proteins via western blot assay. After quantitative assay using bicinchoninic acid (BCA) protein quantification kit (Invitrogen) for serum samples in each group, samples were boiled at $95^{\circ} \mathrm{C}$ water bath for 10 min of degeneration, and the denatured samples were loaded for sodium dodecyl sulfate polyacrylamide gel electrophoresis (SDS-PAGE). Thereafter, the proteins were transferred to the polyvinylidene fluoride (PVDF) membrane. After $2 \mathrm{~h}$ of blocking, the target stripe was then cut off for incubation using the primary antibodies (1:1000) against Drp1, IL-6, TNF- $\alpha$ and MCP-1 overnight at $4^{\circ} \mathrm{C}$. With Tris-buffer saline + Tween-20 (TBST), the membrane was washed 3 times, and then compressed in the ECL solution (mixture of solution A and solution in 1:1) in the dark, during which the compression time was ascertained according to the fluorescent strength of protein stripes. Then, color development and fixation were performed for the membrane, and the stripe was then scanned and was analysed for gray value using ImageJ software.

Statistical analysis. In this study, the data are presented as mean \pm standard deviation, and processed using SPSS 19.0 (SPSS Inc., Chicago, IL, USA) for analysis.t-test was performed for intergroup comparison, and analysis of variance (ANOVA) for comparisons among several groups. After homogeneity test of variance, Bonferronic method was adopted for pairwise comparison if the variance was equal; otherwise the Welch method was adopted. For multiple comparisons, Dunnett's T3 method was used. $\mathrm{P}<0.05$ was considered to indicate a statistically significant difference.

\section{Results}

Variations in blood pressure of rats. Before and at 4 weeks after drug administration of rats in each group, we detected variations in blood pressure, and the results are shown in Fig. 1. Before drug administration, the blood pressure of rats in the SHR group and the MD group was significantly higher than that in the $\mathrm{C}$ group $(\mathrm{P}<0.01)$; after 4 weeks of drug administration, a significant decrease was identified in blood pressure in the rats of the MD group compared with the SHR group $(\mathrm{P}<0.01)$.

CRP level in serum. RIA was applied to detect the concentration of CRP in serum, and the results are shown in Fig. 2. The concentration of CRP in serum of rats in the $\mathrm{C}$ group was significantly lower than those in the SHR group and the MD group $(\mathrm{P}<0.01)$; the level of CRP in serum of rats in the MD group was lower than that in the SHR group, and the difference had statistical significance $(\mathrm{P}<0.01)$.

Variations in media of thoracic aorta of rats in each group. Under the microscope, we observed the variations in media of thoracic aorta of rats in each group that received the hematoxylin and eosin (H\&E) staining, and the results are shown in Fig. 3. The smooth muscle cells in media of thoracic aorta of rats in the $\mathrm{C}$ group were in alignment; in the SHR group, the smooth muscle cells were in malalignment with the increase in stained cells and thickened media; but after the administration of Mdivi-1, compared with the rats in the SHR group, the smooth muscle cells of thoracic aorta in rats in the MD group 


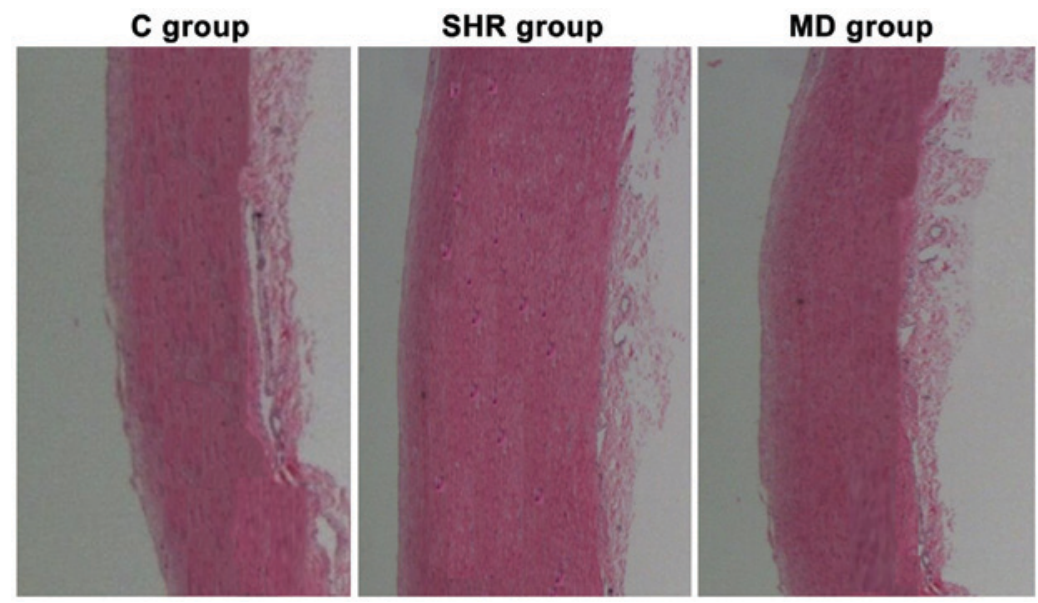

Figure 3. Detecting the variation in media of thoracic aorta of rats in each group using hematoxylin and eosin (H\&E) staining. Compared with the C group, the media of thoracic aorta of rats in the spontaneous hypertension rat (SHR) group are significantly thickened; while the media in the MD group are attenuated.

A

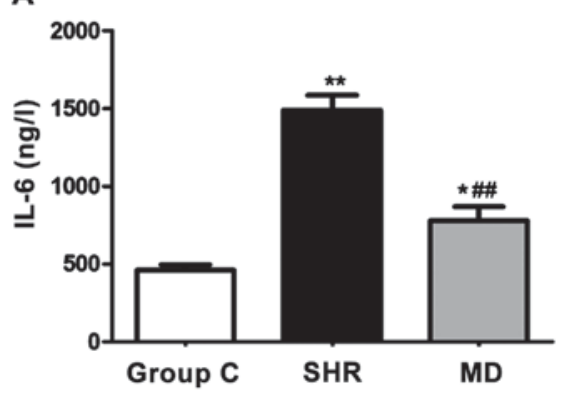

B

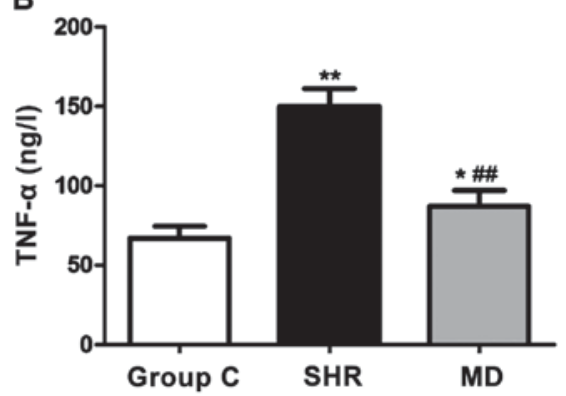

Figure 4. Detection of the expression levels of inflammatory factors via ELISA. (A) The level of IL-6. (B) The level of TNF- $\alpha$. Compared with the C group, the levels of IL- 6 and TNF- $\alpha$ of rats in the SHR group and the MD group are significantly elevated, and the differences have statistical significance ( ${ }^{* *} \mathrm{P}<0.01$, $\left.{ }^{*} \mathrm{P}<0.05\right)$; the levels of IL-6 and TNF- $\alpha$ in serum of rats in the MD group are lower than those in the MD group $\left.{ }^{\# \# ~} \mathrm{P}<0.01\right)$.

were in regular alignment, and attenuation was observed in the thickened media in thoracic aorta.

Detection of the expression levels of inflammatory factors via ELISA. With the ELISA kit, we detected the expression levels of IL- 6 and TNF- $\alpha$ of rats in each group, and the results are shown in Fig. 4. Compared with the $\mathrm{C}$ group, the levels of IL-6 and TNF- $\alpha$ of rats in the SHR group and the MD group were significantly elevated, and the differences had statistical significance $(\mathrm{P}<0.01, \mathrm{P}<0.05)$; the levels of IL-6 and TNF- $\alpha$ in serum of rats in the MD group were lower than those in the MD group $(\mathrm{P}<0.01)$.

Detection of expression levels of Drpl and MCP-1 via semiquantitative PCR. We detected the mRNA expression levels of Drp1 and MCP-1 via semi-quantitative PCR, and the results are shown in Fig. 5. Compared with the $\mathrm{C}$ group, the mRNA expression levels of Drp1 and MCP-1 of rats in the SHR group and the MD group were significantly elevated, and the differences had statistical significance $(\mathrm{P}<0.01)$; in comparison with the SHR group, the mRNA expression levels of Drp1 and MCP-1 of rats in the MD group were decreased $(\mathrm{P}<0.01)$.

Detection of expression levels of proteins via western blot assay. The protein expression levels of Drp1, MCP-1, IL-6 and TNF- $\alpha$ were detected through western blot assay, and the results are shown in Fig. 6. Compared with the $\mathrm{C}$ group, the protein expression levels of Drp1, MCP-1, IL- 6 and TNF- $\alpha$ of rats in the SHR group and the MD group were significantly increased $(\mathrm{P}<0.01, \mathrm{P}<0.05)$; in comparison with the SHR group, significant decreases were identified in the protein expression levels of Drp1, MCP-1, IL-6 and TNF- $\alpha$ of rats in the MD group, and the differences had statistical significance $(\mathrm{P}<0.01)$.

\section{Discussion}

Hypertension patients usually suffer from vascular lesions, and long-term chronic hypertension will generate variations in vascular structure and function, thereby causing vascular remodeling, and increment in thickness of vascular wall, in which the vascular media are thickened and invade into the lumen, clinically called as the vascular hypertrophic remodeling (10). Vascular lesions are the major factors inducing coronary heart disease and stroke, severely affecting the life quality and clinical efficacy of patients (11). The study of Hua (12) revealed that the inflammation of vascular endothelium is one of the key causes for vascular remodeling, and the dysfunction in vascular endothelial cells will lead to increment in release of inflammatory factors. Castellano et al (13) reduced the vascular inflammatory responses in thoracic aorta in rat, which could effectively ameliorate the injuries of 

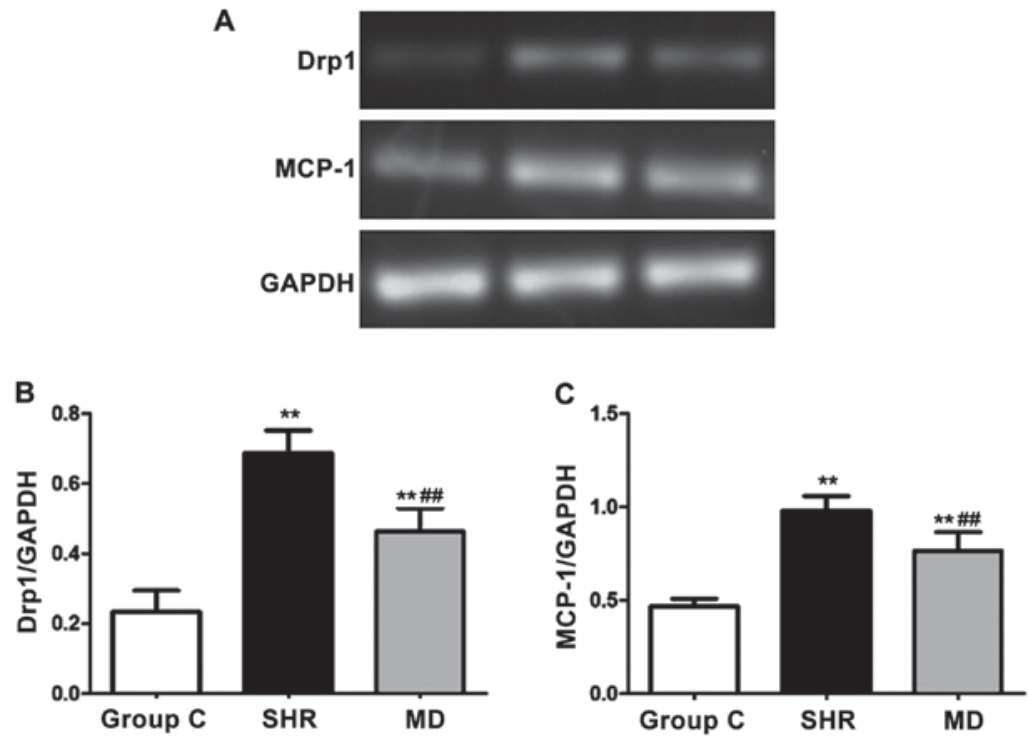

Figure 5. Detection of expression levels of Drp1 and MCP-1 via semi-quantitative PCR. Compared with the C group, the mRNA expression levels of Drp1 and MCP-1 of rats in the SHR group and the MD group are significantly elevated, and the differences have statistical significance $\left({ }^{* *} \mathrm{P}<0.01\right)$; in comparison with the SHR group, the mRNA expression levels of Drp1 and MCP-1 of rats in the MD group are decreased $\left({ }^{\# \#} \mathrm{P}<0.01\right)$.

A

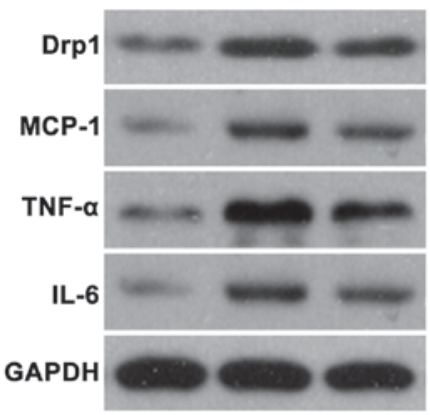

C

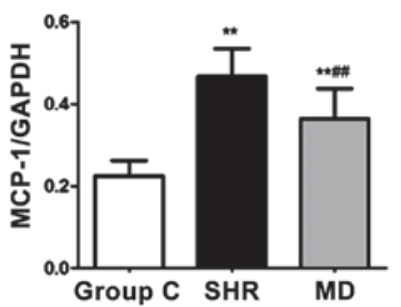

D

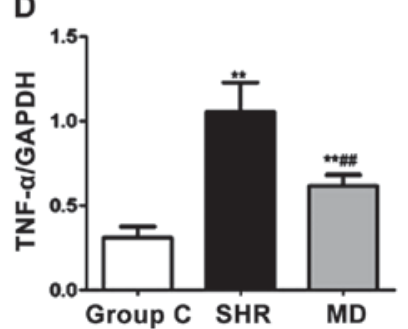

B

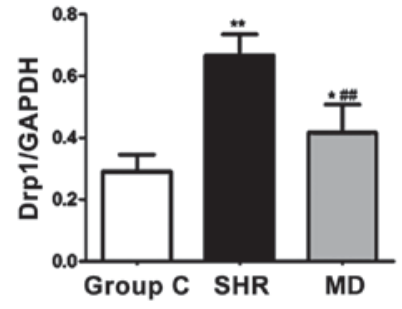

$E$

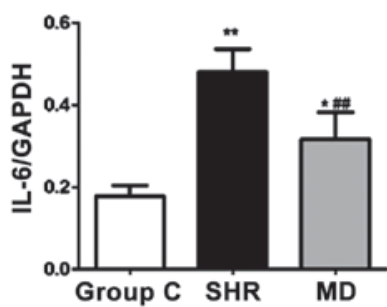

Figure 6. Detection of expression levels of proteins via western blotting assay. Compared with the C group, the protein expression levels of Drp1, MCP-1, IL-6 and TNF- $\alpha$ of rats in the spontaneous hypertension rat (SHR) group and the MD group are significantly increased ("P $\left.<0.05,{ }^{* *} \mathrm{P}<0.01\right)$; in comparison with the SHR group, significant decreases are identified in the protein expression levels of Drp1, MCP-1, IL- 6 and TNF- $\alpha$ of rats in the MD group, and the differences have statistical significance $\left.{ }^{(\#)} \mathrm{P}<0.01\right)$.

hypertension to vessels, and improve the vascular remodeling. The pathologic conditions of inflammation include the activation of pro-inflammatory transcription factor and damage to the hypertension vessels (14).

Currently, researchers, through multiple aspects of mitochondrial dysfunction, have confirmed that in patients with hypertension complicated with vascular lesions usually suffer metabolic dysfunction, damage to energy generation and excessive production of reactive oxygen species in mitochondria, which are of great significance for vascular lesions in hypertension (15). Research has shown that Drp1-mediated mitochondrial division is associated with cell apoptosis and necrosis, and decreasing the mitochondrial division induced by Drp1 can protect the vessels (16).

In this study, SHR rats were used for investigating the correlation between Drp1 in the vascular endothelial cells and inflammatory factors. SHR rat is a kind of spontaneous hypertension rat model developed from Wistar rat in 1963 with the features of stability and high incidence rate of hypertension. At 16 weeks, significant elevation in blood pressure can be seen in SHR rats, thereby inducing hypertension. Besides, the pathogenesis and post-onset vascular and systemic pathologic changes of SHR rats are coincident with the spontaneous hypertension in human beings, and SHR rats have been recognized 
as the animal model that can mimic the onset of spontaneous hypertension. In addition, SHR rats are usually used for fundamental research on spontaneous hypertension and screening the anti-hypertensive drugs (17).

In this study, we found that in SHR rats, the expression of Drp1 was significantly elevated in the vascular endothelial cells, the media of thoracic aorta were thickened, and the administration of Mdivi-1 for 4 weeks could effectively decrease the blood pressure in SHR rats, reduce the expression of Drp1 to a certain extent, and attenuate the thickness of media in thoracic aorta caused by the hypertension. All these results suggested that hypertension can increase the expression of Drp1 in vascular endothelial cells, while suppression on expression of Drpl can decrease the blood pressure and protect the inner wall of the vessel. Disorder in functions of vascular endothelial cells can affect the generation and release of the substances that can invigorate the circulation of blood, thereby attenuating the vasodilatation, and enhancing the vasoconstriction. This will contribute to the increase in hypertension and thickness of vascular wall, which in turn will reduce the vascular compliance, narrow down the lumen, increase the blood resistance and augment the blood pressure $(18,19)$. The expression levels of inflammatory factors in serum of rats in each group were detected via ELISA and western blot assay, and we found that the levels of IL- 6 and TNF- $\alpha$ in serum of SHR rat were remarkably elevated, and that the mRNA and protein expression levels of MCP-1 and Drp1 were increased, but after the administration of inhibitor, the expression levels of IL-6, TNF- $\alpha$ and MCP-1 in serum of SHR rat could be effectively decreased. High expression of Drp1 in vascular endothelium in hypertension can induce the pro-inflammatory factors, and further damage the vessels (20). Through inhibition on Drp1 expression, the expression of inflammatory factors can be effectively decreased, and, therefore, the vessels are protected. In this study, we did not investigate the mechanism that Drp1 affected in the release of inflammatory factors in vessels, but the relevant mechanism could better explain the effect and the mechanism of Drp1, which is one of the shortages in this study. We anticipate to perform more studies on this mechanism.

In the vascular endothelium of hypertension, Drp1 is highly expressed, and the excessive production of inflammatory factors is also induced, which can lead to the damage to vascular endothelium and increment in thickness of media in vascular wall. Mdivi-1 can decrease the expression of Drp1, thereby ameliorating the inflammatory responses and protecting the vessels in SHR rats.

\section{Acknowledgements}

Not applicable.

\section{Funding}

This study was supported by the Foundation of Science and Technology Bureau of Guiyang [zhukehetong (20151001) she60hao].

\section{Availability of data and materials}

The datasets used and/or analyzed during the present study are available from the corresponding author on reasonable request.

\section{Authors' contributions}

XinL contributed to the conception and design of the study. HT collected the data and revised the manuscript for important intellectual content. XiaL and QW analyzed and interpreted the data, and drafted the manuscript. All authors read and approved the final manuscript.

\section{Ethics approval and consent to participate}

The study was approved by the Ethics Committee of Guizhou Provincial People's Hospital (Guiyang, China).

\section{Consent for publication}

Not applicable.

\section{Competing interests}

The authors declare that they have no competing interests.

\section{References}

1. Song CL, Zhang X, Liu YK, Yue WW and Wu H: Heart rate turbulence in masked hypertension and white-coat hypertension. Eur Rev Med Pharmacol Sci 19: 1457-1460, 2015.

2. Misárková E, Behuliak M, Bencze M and Zicha J: Excitationcontraction coupling and excitation-transcription coupling in blood vessels: Their possible interactions in hypertensive vascular remodeling. Physiol Res 65: 173-191, 2016.

3. Kai H, Kudo H, Takayama N, Yasuoka S, Aoki Y and Imaizumi T: Molecular mechanism of aggravation of hypertensive organ damages by short-term blood pressure variability. Curr Hypertens Rev 10: 125-133, 2014.

4. Rubattu S, Stanzione R and Volpe M: Mitochondrial dysfunction contributes to hypertensive target organ damage: lessons from an animal model of human disease. Oxid Med Cell Longev 2016: 1067801, 2016.

5. Zhang J, Fallahzadeh MK and McCullough PA: Aging male spontaneously hypertensive rat as an animal model for the evaluation of the interplay between contrast-induced acute kidney injury and cardiorenal syndrome in humans. Cardiorenal Med 7: 1-10, 2016.

6. Ahmeda $\mathrm{AF}$ and Alzoghaibi $\mathrm{M}$ : Factors regulating the renal circulation in spontaneously hypertensive rats. Saudi J Biol Sci 23: 441-451, 2016.

7. Hu C, Huang Y and Li L: Drp1-dependent mitochondrial fission plays critical roles in physiological and pathological progresses in mammals. Int J Mol Sci 18: 144, 2017.

8. Huang P, Sun Y, Yang J and Chen S: Dynamin-related protein 1 (Drpl) promotes structural intermediates of membrane division. J Biol Chem 206: 26-32, 2016.

9. Tanwar DK, Parker DJ, Gupta P, Spurlock B, Alvarez RD, Basu MK and Mitra K: Crosstalk between the mitochondrial fission protein, Drp1, and the cell cycle is identified across various cancer types and can impact survival of epithelial ovarian cancer patients. Oncotarget 7: 60021-60037, 2016.

10. Marsboom G, Toth PT, Ryan JJ, Hong Z, Wu X, Fang YH, Thenappan T, Piao L, Zhang HJ, Pogoriler J, et al: Dynaminrelated protein 1-mediated mitochondrial mitotic fission permits hyperproliferation of vascular smooth muscle cells and offers a novel therapeutic target in pulmonary hypertension. Circ Res 110: 1484-1497, 2012.

11. Zhang W, Li R, Liu S and Zhang J: Novel role for the regulation of mitochondrial fission by HIF- $1 \alpha$ in the control of smooth muscle remodeling and progression of pulmonary hypertension. BioMed Res Int 4: 577-590, 2017.

12. Hua JN: Inflammation and hypertension: the interplay of interleukin-6, dietary sodium and the renin-angiotensin system in humans. PLoS One 36: 7591-7598, 2014.

13. Castellano G, Melchiorre R and Loverre A: Interleukin-6 underlies angiotensin II-induced hypertension and chronic renal damage. Am J Pathol 8: 435-447, 2016. 
14. Park SY, Shrestha S, Youn YJ, Kim JK and Kim SY: Deletion of interleukin-6 prevents cardiac inflammation, fibrosis and dysfunction without affecting blood pressure in angiotensin II-high salt-induced hypertension. Asian Pac J Trop Med 9: 2-9, 2016.

15. Brands MW, Banes-Berceli AKL, Inscho EW, Al-Azawi H, Allen AJ and Labazi H: Interleukin-6 knockout prevents angiotensin II hypertension: Role of renal vasoconstriction and JAK2/ STAT3 activation. Hypertension 56: 879-884, 2010.

16. Wang Y,Zhu M, Xu H, Cui L, Liu W, Wang X, Shen S and Wang DH: Role of the monocyte chemoattractant protein-1/C-C chemokine receptor 2 signaling pathway in transient receptor potential vanilloid type 1 ablation-induced renal injury in salt-sensitive hypertension. Exp Biol Med (Maywood) 240: 1223-1234, 2015.

17. Li Y and Liu S: Effect of the antihypertensive drug enalapril on oxidative stress markers and antioxidant enzymes in kidney of spontaneously hypertensive rat. Med Sci Monit 53: 25-32, 2016.
18. Batinic-Haberle I, Tovmasyan A and Emily RH: Blockade of CCR2 reduces macrophage influx and development of chronic renal damage in murine renovascular hypertension. Antioxid Redox Signal 30: 266-271, 2014.

19. Zhang L, Gan W and An G: IL-10 supplementation increases Tregs and decreases hypertension in the RUPP rat model of preeclampsia. Neural Regen Res 20: 97-110, 2016.

20. Nevers T, Kalkunte $S$ and Sharma S: Uterine regulatory T cells, IL-10 and hypertension. Am J Reprod Immunol 66 (Suppl 1): 88-92, 2011.

This work is licensed under a Creative Commons Attribution-NonCommercial-NoDerivatives 4.0 International (CC BY-NC-ND 4.0) License. 